\title{
Bone marrow stem cell damage after three different chemotherapy regimens for advanced Hodgkin's lymphoma
}

\author{
PAOLO G. GOBBI ${ }^{1}$, FRANCESCO VALENTINO ${ }^{1}$, MARCO DANOVA ${ }^{1}$, FORTUNATO MORABITO ${ }^{2}$, \\ BIANCA ROVATI ${ }^{1}$, CATERINA MAMMI $^{3}$, MASSIMO GENTILE $^{2}$, FRANCESCO MERLI ${ }^{4}$, \\ CATERINA STELITANO ${ }^{5}$, STEFANO LUMINARI ${ }^{3}$, GIANNI QUINTANA ${ }^{6}$, \\ EMILIO IANNITTO $^{7}$, MAURA BRUGIATELLI $^{8}$ and MASSIMO FEDERICO ${ }^{3 *}$ \\ ${ }^{1}$ Clinica Medica I, Università di Pavia, IRCCS Policlinico S. Matteo, Pavia; ${ }^{2}$ U.O.C. di Ematologia, \\ Azienda Ospedaliera, Cosenza; ${ }^{3}$ Oncologia Medica, Policlinico e Università di Modena; \\ ${ }^{4}$ Servizio di Ematologia, Arcispedale 'S. Anna', Reggio Emilia; ${ }^{5}$ Divisione di Ematologia, \\ Ospedali Riuniti, Reggio Calabria; ${ }^{6}$ Divisione di Ematologia, Ospedale ‘A. Perrino', Brindisi; \\ ${ }^{7}$ Cattedra e Divisione di Ematologia con Trapianto di Midollo Osseo, Policlinico e Università \\ di Palermo; ${ }^{8}$ Divisione di Ematologia, Ospedale 'Papardo', Messina-Sperone, Italy
}

Received December 5, 2008; Accepted January 19, 2009

DOI: 10.3892/or_00000320

\begin{abstract}
The aim of this study was to evaluate the apoptotic damage to bone marrow cells caused by three chemotherapy regimens for advanced Hodgkin's lymphoma, ABVD, COPPEBVCAD and BEACOPP, which were randomly administered in the HD 2000 GISL trial. Bone marrow mononuclear cells (BMMCs) stained with anti-CD34 antibody and Annexin $\mathrm{V}$, were evaluated by flow cytometry before starting chemotherapy, 30 days after completing chemotherapy and after 6 months. Results are expressed as the percentages of BMMCs positive to anti-CD34, to Annexin V or to both. Fourteen patients treated with ABVD, 11 with COPPEBVCAD and 13 with BEACOPP were evaluated before and 30 days after treatment. Late assessments were made in 6,7 and 8 of them, respectively. No differences were found among the pretherapeutic flow cytometry findings in relation to the staging characteristics (marrow involvement included). All the regimens increased the apoptotic fraction of the whole mononuclear bone marrow cells (COPPEBVCAD did so significantly) and increased the CD34+ compartment (with significant early differences after $\mathrm{ABVD}$ and BEACOPP, tending to late persistence for ABVD, only). All the regimens increased the apoptotic $\mathrm{CD} 34^{+}$cells within the whole BMMC population (significantly after
\end{abstract}

Correspondence to: Professor Paolo G. Gobbi, Clinica Medica I, Università di Pavia, Fondazione IRCCS Policlinico S. Matteo, P. le Golgi no. 2, 27100 Pavia, Italy

E-mail: gobbipg@smatteo.pv.it

*On behalf of the Gruppo Italiano Studio Linfomi (GISL)

Key words: Annexin V, stem cells, chemotherapy, Hodgkin
BEACOPP), although with a general trend to decrease in their percentage within the $\mathrm{CD} 34^{+}$compartment over time, even after the most dose-dense regimens. Based on the variations induced in the apoptotic fraction of all mononuclear and $\mathrm{CD} 34^{+}$cells, $\mathrm{ABVD}$ was the least toxic regimen and COPPEBVCAD the most toxic one.

\section{Introduction}

Combination chemotherapy has been the cornerstone of the treatment of advanced Hodgkin's lymphoma for several decades. Initial optimism generated from the dramatic results achieved with mechlorethamine, vincristine, procarbazine, and prednisolone (MOPP) was later tempered by two growing realizations: first, that further improvements in clinical results would be slow, of reduced impact, and hard-earned; secondly, that despite prolonged remission and cure, serious late effects of chemotherapy could be an intolerable counterbalance of clinical results (1). So, when in 2000 the Gruppo Italiano per lo Studio dei Linfomi (GISL) designed a randomized trial comparing three different chemotherapy regimens (2), it also planned, besides the mandatory record of early and late side effects, some parallel investigations aimed at an early and direct evaluation of the effects on the stem cell compartment potentially responsible for persistent marrow damage and the consequent defective ability to mobilize progenitor cells.

Such damage might interfere with the feasibility of autologous bone marrow transplantation (AuBMT), a procedure that might be needed as part of the salvage treatment for resistant or relapsing cases (3). Since the slow improvement of clinical responses achieved over the last 20 years has been reached through intensification of drug doses and resulting toxicity, it is possible that a variable part of the improved response produced by particularly intense and aggressive chemotherapy might be paid for by a reduced possibility of 
rescue with transplantation procedures if these become necessary. It might, therefore, be questioned whether it is better to use a less myelotoxic, though not highly performing, regimen which leaves a good possibility of AuBMT or to achieve the maximum early clinical response even at the risk of compromising the ability to mobilize adequate amounts of stem cells in the case of resistance or relapse. In order to investigate this issue, we assessed the grade of apoptosis caused to BMMCs by three drug regimens currently used in the treatment of patient with advanced-stage Hodgkin's lymphoma: ABVD (4) (doxorubicin, bleomycin, vinblastine and dacarbazine), COPPEBVCAD (a minimal variant of MOPPEBVCAD (5) in which cyclophosphamide replaces mechlorethamine and other drugs, vincristine, procarbazine, prednisone, epidoxorubicin, bleomycin, vinblastine, lomustine, melphalan and vindesine, remain unmodified) and BEACOPP (6) (bleomycin, etoposide, doxorubicin, cyclophosphamide, vincristine, procarbazine and prednisone). Apoptosis was evaluated through the high affinity of Annexin $\mathrm{V}$ for phosphatidylserine, a cell membrane phospholipid which is early exposed on the external surface at the beginning of the apoptotic process.

\section{Materials and methods}

Patients and treatments. The population of the study was an unselected sample of the larger series randomized in the HD 2000 GISL multicenter trial between 2000 and 2006. In this study treatment consisted of six courses of one of the three multiple-drug chemotherapy regimens - ABVD, COPPEBVCAD and BEACOPP- followed by optional radiotherapy, which had to be delivered to no more than two distinct areas corresponding to either bulky masses at diagnosis or incompletely remitting lesions after chemotherapy. The schedules of the randomized regimens are detailed in Table I. The cumulative dose of radiotherapy had to be 30 Gy.

The proposed object was to study at least ten patients in each of the randomization arms. Patients who gave their written consent underwent bone marrow aspiration 1-3 days before the start of chemotherapy (assessment 1), 30 days after stopping chemotherapy (assessment 2) and 6 months after the end of chemotherapy (assessment 3), irrespective of whether the optional brief program of irradiation was delivered after chemotherapy. The marrow samples were collected in test-tubes, sealed in containers at a constant temperature and sent by a quick courier service to reach the central evaluation laboratory within no more than $24 \mathrm{~h}$. The patients were admitted to the study based simply on their willingness to undergo the procedures and the agreement of their GISL treatment center to co-operate in this study; some willing patients from co-operating studies were, however, excluded because of clinical or technical circumstances potentially interfering with the collection or analysis of their samples (for example, clinical conditions requiring immediate treatment, proximity of bank holidays, casual temporary inability of the central laboratory, accidental damage of the sample during transport). In all, 14 patients treated with ABVD, 11 with COPPEBVCAD and 13 with BEACOPP underwent assessments 1 and 2 while 6,7 and 8 , respectively had assessment 3 . There were no selection biases affecting the three groups of patients, as demonstrated by the clinical characteristics reported in Table II.

Samples. Fresh bone marrow was obtained from the posterior superior iliac spine. Two milliliters of marrow were sampled from the first vigorous aspiration into a heparinized syringe, and put into a plastic test-tube with a watertight cap. The samples were then sent, without delay and protected from thermal shock, to the central laboratory where they underwent cell death assays.

Cell death assays. BMMCs were isolated by density gradient centrifugation over Ficoll-Paque $(\mathrm{d}=1.077 \mathrm{~g} / \mathrm{ml}$; Pharmacia Biotech) and washed twice in phosphatebuffered saline (PBS, Sigma). A total of $10^{6}$ cells per sample were used. Cells were incubated with phycoerythrin (PE)conjugated and anti-CD34 monoclonal antibody (Mab, Clone 8G12, Anti-HPCA-2, IgG1; Becton-Dickinson) for $20 \mathrm{~min}$ at room temperature in the dark and then washed twice in PBS. Pelleted cells were resuspended in $100 \mu \mathrm{l}$ binding buffer (10 mmol/l Hepes/NaOH, pH 7.4, 140 mmo/l NaCl, $2.5 \mathrm{mmo} / \mathrm{C} \mathrm{CaCl}_{2}$; Bender Medsystems, Boehringer Ingelheim) and incubated with $10 \mu \mathrm{l}$ fluorescein isothiocyanate (FITC)conjugated Annexin V (Bender Medsystems, Boehringer Ingelheim) for $10 \mathrm{~min}$ at room temperature in the dark. Cells were then resuspended in $400 \mu \mathrm{l}$ binding buffer prior to flow cytometric analysis. Negative controls included MNC incubated with neither CD34-PE Mab nor Annexin-V-FITC and cells incubated with CD34-PE Mab only.

Two-color analysis of Annexin V within CD34+ cell populations was performed using an EPICS XL flow cytometer (Coulter). Data were obtained in list mode, acquiring at least $2 \times 10^{6}$ events per sample and/or at least $10^{4}$ events within the CD34+ cell gate. The analysis of apoptosis was based on gating subpopulations of $\mathrm{CD} 34^{+}$cells by forward scatter vs. side scatter as well as side scatter vs. fluorescence 2 . The percentage of apoptotic cells was determined by comparing the fluorescence distribution histogram of positively stained cells to that of cells to which no Annexin V had been added (Fig. 1).

The following four evaluations were made on each sample: per cent of BMMCs positive to FITC-Annexin V $\left(A n n^{+}\right)$, including both myelopoietic and immunopoietic component; per cent of BMMCs positive to CD34-PE Mab $\left(\mathrm{CD} 34^{+}\right)$, which corresponds to the myelopoietic compartment only; per cent of BMMCs positive to both CD34-PE Mab and FITC-Annexin $\mathrm{V}\left(34^{+} \mathrm{A}^{+}\right)$, wich gives information about the death fraction among the myelopoietic cells related to the whole BMMCs; per cent of all $\mathrm{CD} 34^{+}$cells that were also positive to Annexin $\mathrm{V}\left(\mathrm{A}^{+} / 34^{+}\right)$, which reflects the death fraction within the hemopoietic stem cells. This parameter was considered to evaluate the apoptotic fraction of the $\mathrm{CD} 4^{+}$cells independent of the contemporary possible variation of apoptosis among the BMMCs.

Statistical analysis. Given that the data were not normally distributed, comparisons were tested using Wilcoxon's rank sum test and were performed among groups two by two (7). The data are graphically represented in Figs. 1-4 by means of box plots that show the 10th, 25th, 50th (i.e., the median), 75 th 
Table I. Drug doses and time schedules of the three chemotherapy regimens investigated in the study.

\begin{tabular}{|c|c|c|c|}
\hline Drug & Dose $\left(\mathrm{mg} / \mathrm{m}^{2}\right)$ & Route & Days \\
\hline \multicolumn{4}{|l|}{$\mathrm{ABVD}^{\mathrm{a}}$} \\
\hline Doxorubicin & 25 & i.v. & 1.15 \\
\hline Bleomycin & 5 & i.v. & 1.15 \\
\hline Vinblastine & 6 & i.v. & 115 \\
\hline Dacarbazine & 375 & i.v. & 1.15 \\
\hline \multicolumn{4}{|l|}{ COPPEBVCAD } \\
\hline Cyclophosphamide & 650 & i.v. & 1 (cycles 1,3 and 5 , only) \\
\hline Lomustine (CCNU) & 100 & p.o & 1 (cycles 2,4 and 6, only) \\
\hline Vindesine & 3 & i.v. & 1 \\
\hline Melphalan & 6 & p.o. & $1-3$ \\
\hline Prednisone & 40 & p.o. & $1-14$ \\
\hline Epidoxorubicin & 40 & i.v. & 8 \\
\hline Vincristine & 1.4 & i.v. & 8 \\
\hline Procarbazine & 100 & p.o. & $8-14$ \\
\hline Vinblastine & 6 & i.v. & 15 \\
\hline Bleomycin & 10 & i.v. & 15 \\
\hline \multicolumn{4}{|l|}{$\mathrm{BEACOPP}^{\mathrm{b}}$} \\
\hline Cyclophosphamide & $650(1200)$ & i.v. & 1 \\
\hline Doxorubicin & $25(35)$ & i.v. & 1 \\
\hline Etoposide & $100(200)$ & i.v. & $1-3$ \\
\hline Procarbazine & 100 & p.o. & $1-7$ \\
\hline Vincristine $^{c}$ & 1.4 & i.v. & 8 \\
\hline Bleomycin & 10 & i.v. & 8 \\
\hline Prednisone $^{\mathrm{d}}$ & 40 & p.o. & $1-14$ \\
\hline
\end{tabular}

Six cycles were planned for all the regimens. ${ }^{a}$ Cycles were repeated every 28 days; day 29 was the start of a new cycle. ${ }^{b}$ Doses outside brackets refer to the baseline schedule, those inside brackets to the intensified version; cycles 1-4 were intensified, cycles 5-6 baseline; cycles were repeated every 21 days, day 22 being start of a new cycle.

Table II. Clinical characteristics of the patients studied according to the treatment administered.

\begin{tabular}{lccc}
\hline & ABVD & COPPEBVCAD & BEACOPP \\
\hline Number & 14 & 11 & 13 \\
Median age (and range) & $36(20-65)$ & $33(15-61)$ & $36(21-61)$ \\
Male/Female ratio & $5 / 9$ & $5 / 6$ & $6 / 7$ \\
Stage IIB/III/IV & $4 / 7 / 3$ & $2 / 5 / 3$ & $4 / 5 / 4$ \\
Histology: LD vs. others & $2 / 12$ & $3 / 8$ & $3 / 10$ \\
Bulky mass & 8 & 5 & 6 \\
Hb $(\mathrm{g} / \mathrm{dl}$, mean \pm SD) & $11.8 \pm 1.7$ & $11.0 \pm 1.8$ & $11.4 \pm 2.1$ \\
Alb $(\mathrm{g} / \mathrm{l}$, mean \pm SD) & $3.9 \pm 0.7$ & $3.8 \pm 0.5$ & $3.6 \pm 0.7$ \\
LDH (mU/ml, mean \pm SD) & $487 \pm 227$ & $416 \pm 231$ & $481 \pm 282$ \\
WBC $(10 / 1$, mean \pm SD) & $10.3 \pm 5.2$ & $12.7 \pm 6.1$ & $11.4 \pm 5.4$ \\
Ly $(10 / 1$, mean \pm SD) & $1.5 \pm 0.8$ & $2.2 \pm 1.2$ & $1.7 \pm 1.0$ \\
Radiotherapy & 7 & 5 & 6 \\
\hline
\end{tabular}

LD, lymphocyte depletion; Hb, hemoglobin concentration; Alb, serum albumin concentration; LDH, serum lactate dehydrogenase concentration; WBC, white blood cell count; Ly, blood lymphocyte count. 

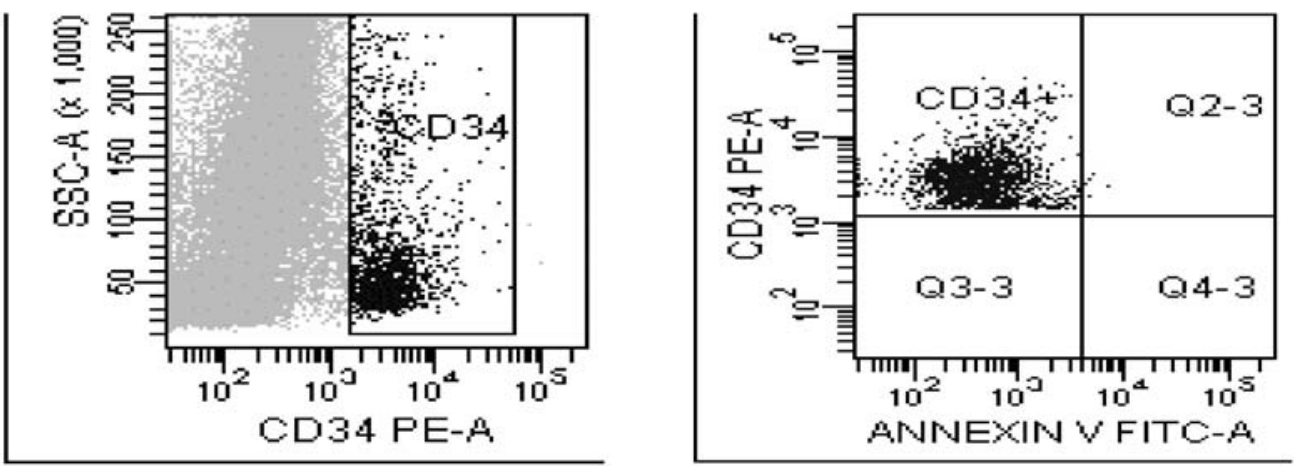

a

b

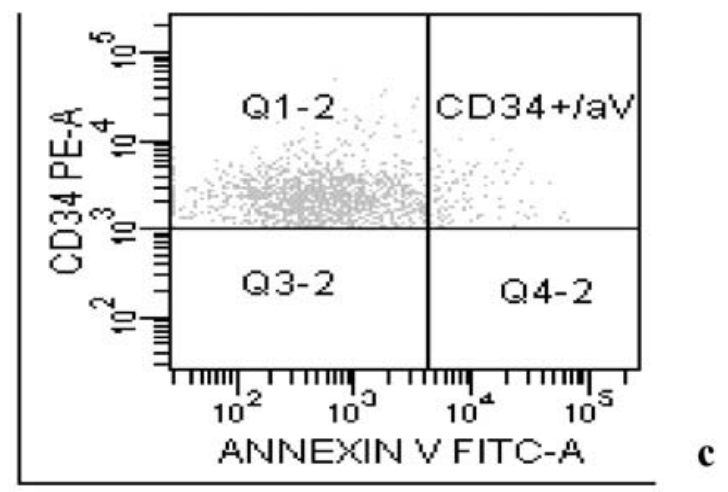

Figure 1. Flow cytometric evaluation of $\mathrm{CD} 34^{+}$cell apoptosis. Analysis was based on gating on $\mathrm{CD} 34^{+}$cell subpopulations side scatter (SSC) vs. fluorescence 2 (CD34PE) (a). Percentage of posivity was determined by comparison of the fluorescence distribution histogram (Annexin V FITC) of positively stained cells (c) to that of cells to which no Annexin V was added (b).

and 90th percentiles of data, with single values above the 90th and below the 10th percentiles plotted as distinct points.

\section{Results}

No differences were found among the distribution of $\mathrm{Ann}^{+}$, $\mathrm{CD}^{2} 4^{+}, 34^{+} \mathrm{A}^{+}$and $\mathrm{A}^{+} / 34^{+}$cells in relation to the main staging characteristics (stage; constitutional symptoms; bulky disease; histology; hemoglobin, serum albumin, and serum lactate dehydrogenase concentrations; marrow involvement) and to the treatment arms.

The proportion of apoptotic cells within the whole population of BMMCs was not substantially increased after either ABVD or BEACOPP treatment (Fig. 2), while there was a clear rise after COPPEBVCAD, both early and late after the end of therapy. Fig. 3 illustrates the percentage variations of the $\mathrm{CD}^{+} 4^{+}$cells according to treatment arm. There were increases in $\mathrm{CD} 34^{+}$cells early after all treatments (significant in the ABVD arm), with a trend to higher and significant late values after both ABVD and BEACOPP.

The percentage of the cells simultaneously positive to CD34-PE Mab and FITC-Annexin V (i.e., the apoptotic stem cells) related to the whole BMMC compartment increased after therapy, whichever regimen was administered (Fig. 4). This increase was more evident earlier than later after all treatments; the percentage of apoptotic stem cells was significantly highest after BEACOPP treatment and less high after the ABVD and COPPEBVCAD regimens.

Fig. 5 illustrates the variations of the apoptotic cell fraction within the stem cell population. The percentages of these $\mathrm{Ann}^{+}$cells within the whole CD $34^{+}$compartment tended to decrease after chemotherapy (i.e., the increase of all the progenitor cells is higher than that of their apoptotic quote) except for COPPEBVCAD, which showed persisting higher percentages of apoptotic cells 30 days after the end of treatment. Though these variations are not statistically significant, the distribution of the data, and their median values, provides some evidence of a more considerable and progressive decrease after BEACOPP than after the other regimens.

No substantial differences were found in the results of the assays performed 6 months after chemotherapy according to whether the patients had received radiotherapy or not.

\section{Discussion}

Apoptosis is the primary mechanism by which radiation and most chemotherapeutic agents induce tumor cell death. This effect is not selective, involving both normal and malignant cells, and depends on whether each individual cell is committed to apoptosis in response to chemotherapy and/or radiotherapy (8). Radiotherapy has progressively disappeared from firstline treatment for Hodgkin's lymphoma and multiple-drug chemotherapy now plays the pivotal role in the management of this disease, leaving irradiation only a selected, limited and complementary function. Moreover, it is becoming clear that in most cases, after failure of primary treatment, highdose chemotherapy followed by AuBMT is significantly more effective than non-transplant salvage therapy, regardless of whether the relapse occurs early or late after primary 

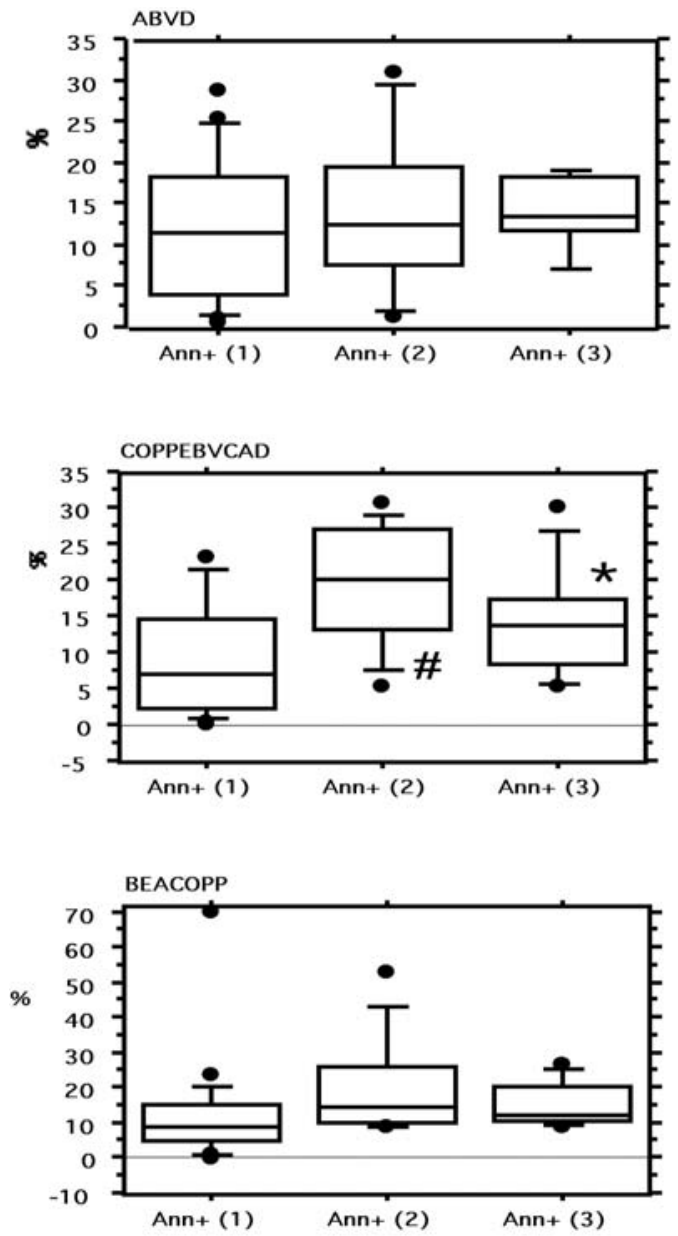

Figure 2. Distribution of the percentages of all the apoptotic cells within the whole bone marrow mononuclear population presented according to chemotherapy arm, and analyzed before starting chemotherapy (1), and at 1 month (2) and 6 months after the end of chemotherapy. The horizontal lines of the box plots show the 10th, 25th, 50th (i.e., the median), 75th and 90th percentiles of the data, whereas single values above the 90th and below the 10th percentiles, if any, are plotted as distinct points. "Statistical differences with $\mathrm{P}<0.05,{ }^{\#}$ Statistical differences with $\mathrm{P}<0.01$.

chemotherapy $(3,9)$. Thus, it is likely that the choice of the first-line chemotherapy should be guided not only by the evidence of its efficacy and consideration of its potential late toxicity, but also by whether it enables AuBMT procedures should the first treatment fail.

For this reason we took the opportunity of studying apoptosis of BMMCs in a limited number of the patients enrolled in the GISL trial HD2000, which had the primary end-point of comparing the efficacy and toxicity of three distinct chemotherapy regimens, ABVD, COPPEBVCAD and BEACOPP, in advanced Hodgkin's lymphoma. The basic assumption regarding the aim of this work was that the treatment inducing the lowest proportion of apoptosis of bone marrow precursors might have a clinical advantage, efficacy and toxicity being equal. It is questionable that the small percentage of the additional early responses achievable with more intensive and toxic chemotherapy may be paid for by difficulty in harvesting bone marrow progenitor cells. In light of this, it seems reasonable that a more complete and correct assessment of the clinical value of a first-line regimen should be coupled to a consideration of the feasibility and,
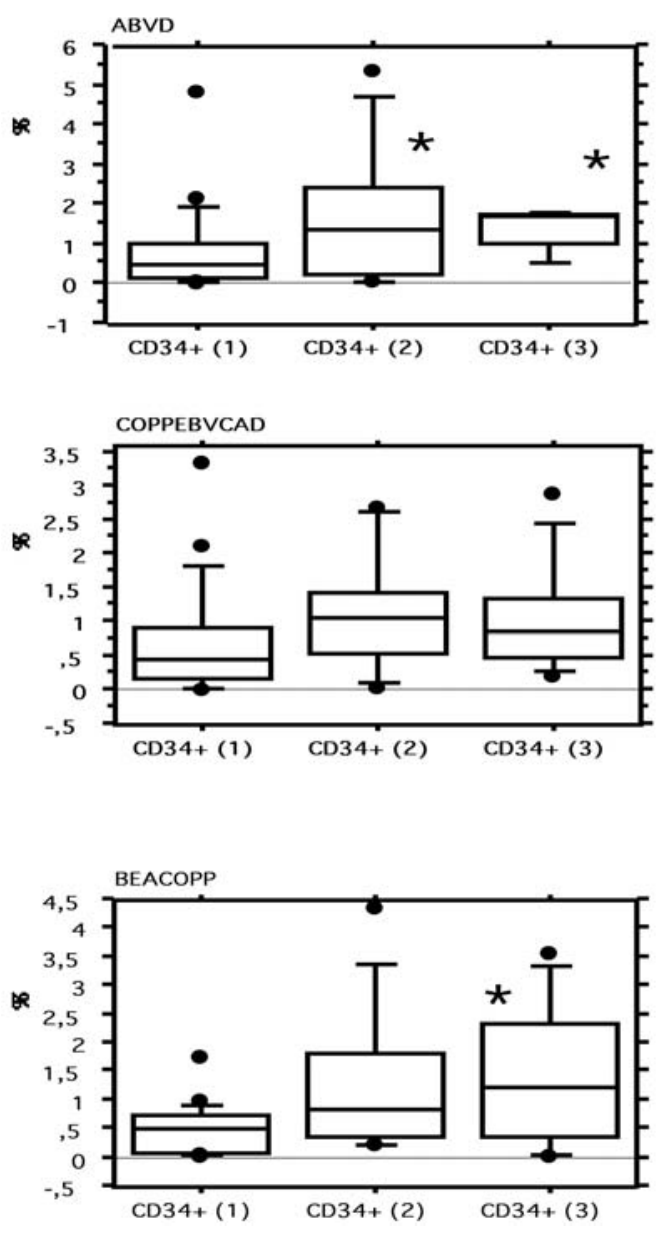

Figure 3. Distribution of the percentages of $\mathrm{CD} 34^{+}$cells within the marrow mononuclear population presented according to chemotherapy arm, and analyzed before starting chemotherapy (1), and at 1 month (2) and 6 months (3) after the end of chemotherapy. Legends as in Fig. 1.

possibly, of the outcome of the planned rescue therapy in the case of primary treatment failure, thus making a global evaluation of the combined treatments. The attention to the current parameters of response and toxicity of first-line treatment alone should, therefore, be expanded to cover the whole combination of first-line and salvage therapy which may need to be offered. The GISL has now closed the recruitment of patients into the HD2000 trial and the very preliminary results have been communicated at the end of $2007(2,10)$. Herein, we present the results on direct, early and late toxicity caused to the stem cell compartment by the three treatment regimens used.

Some comments should be made regarding the results obtained. The results are given as percentages of BMMCs, since absolute counts are subject to a number of biases (quantitative unreliability of any bone marrow aspirate regarding the cell concentration which is actually present in the marrow, together with potential minor technical heterogeneity due to the multicenter basis of the investigation, since it is hard to control for many differences in manual procedures despite the strict instructions given regarding power of aspiration, volume of marrow blood taken in the first aspirate, heparin/blood ratio in the test-tubes). Thus, the percentages given are related to a fixed number of BMMCs, 

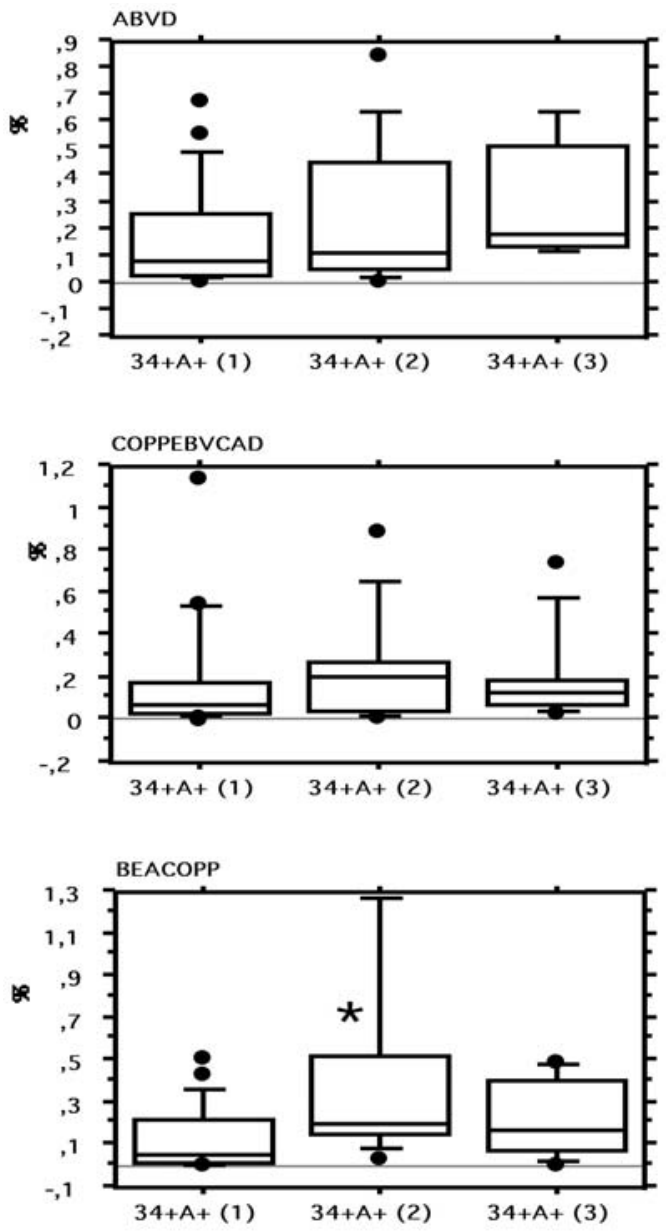

Figure 4. Percentages of the marrow mononuclear cells which are apoptotic presented according to chemotherapy arm, and analyzed before starting chemotherapy (1), and at 1 month (2) and 6 months after the end of chemotherapy. Legends as in Fig. 1.

irrespective of the exact amount of cells actually present in the bone marrow.

The changes in the parameters observed 30 days after the completion of chemotherapy were not biased by radiotherapy, which, when given, was started after the second marrow sample. Neither were the results obtained after 6 months significantly affected by radiotherapy, since the median and distribution of the data from patients who underwent radiotherapy do not differ from those of subjects who did not undergo this treatment. Moreover, the number of sites exposed to radiotherapy, the irradiated volumes, and the cumulative dosages were very limited, so that the influence of radiotherapy on the whole bone marrow compartment could have been expected to be minimal.

The 30-day interval from the end of chemotherapy and the second assays was justified by the need to avoid variations due to immediate toxicity and the consequent spontaneous rapid marrow rescue. To this aim no growth factors were given after the last cycle administered. So, the results at 30 days should not reflect the stimulation induced either by the temporary cytoreduction caused by chemotherapy or by the administration of growth factors. Thus the assays do not directly test the capacity for stem cell mobilization, although it can be assumed that preserving a good amount of vital
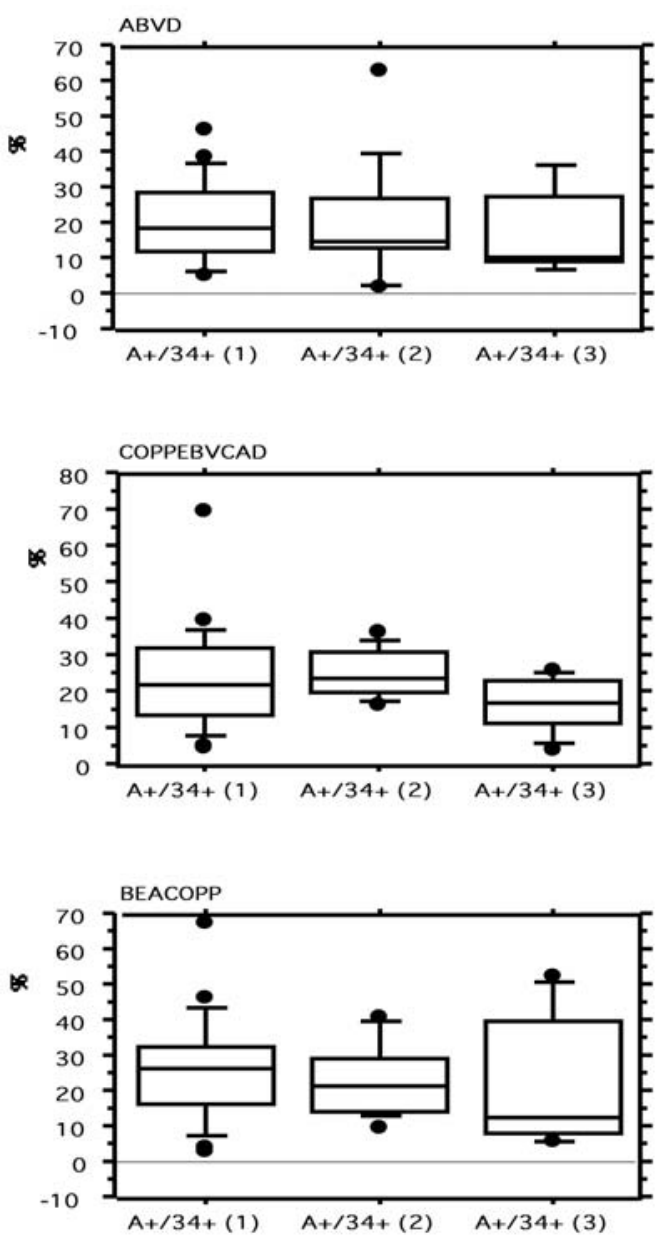

Figure 5. Distribution of the percentage apoptotic fraction of all the CD34 marrow cells presented according to chemotherapy arm, and analyzed before starting chemotherapy (1), and at 1 month (2) and 6 months (3) after the end of chemotherapy. Legends as in Fig. 1.

stem cells is a necessary requirement for satisfactory mobilization and harvesting of $\mathrm{CD} 34^{+}$cells for AuBMT.

ABVD seems to cause the most intense and prolonged increase of the $\mathrm{CD}_{3} 4^{+}$cells, associated with the smallest increase in the whole $\mathrm{Ann}^{+} \mathrm{BMMC}$ compartment. The apoptotic fraction of the CD $34^{+}$cells was not significantly different 6 months after chemotherapy compared with pretreatment values.

COPPEBVCAD led to the lowest increase of $\mathrm{CD}^{2} 4^{+}$cells, combined with the highest and constant rise of $\mathrm{Ann}^{+} \mathrm{BMMCs}$. It can be argued that within this apoptotic compartment, lymphocytes and monocytes increased more than CD34+ cells so that the apoptotic proportion of the $\mathrm{CD} 34^{+}$cells showed a modest non-significant increase after COPPEBVCAD, tending to reduce in the subsequent 6 months.

BEACOPP caused a strong, temporary and statistically significant rise of $\mathrm{CD}^{+} 4^{+}$cells in the first month, together with a moderate, non-significant increase of the $\mathrm{Ann}^{+}$ compartment. Likely, and differently from COPPEBVCAD which presents similar dose density (11), it exerted its toxic effects more on the $\mathrm{CD} 34^{+}$compartment than on other BMMCs and its entire apoptotic fraction significantly increased in the first month, tending to pre-treatment levels after 6 months. 
In conclusion, from the observed modifications of the fractions of $\mathrm{CD}_{34}{ }^{+}$cells and apoptotic cells within the whole BMMC compartment, the ABVD regimen was the least toxic to the bone marrow stem-cell component, while the COPPEBVCAD regimen was the most toxic, causing a generalized and prolonged increase of apoptosis, probably also involving the immunocompetent cells among the MNC population. The presence of alkylating agents and nitrosureas in the COPPEBVCAD regimen might explain this effect. BEACOPP showed an intermediate toxicity: the rise in $\mathrm{CD} 4^{+}$cells that it caused was less prolonged than that following treatment with ABVD, its stimulation of apoptosis was less strong than that of COPPEBVCAD. One month after BEACOPP the apoptotic cell fraction showed the highest increase related to all BMMCs, but a modest decrease within the $\mathrm{CD}_{3} 4^{+}$compartment, with a trend to a progressive reduction over time, similar as that of the other regimens.

\section{Acknowledgements}

We are indebted to Dr Rachel Stenner for her careful revision of the English of this paper. Supported in part by grants from the Ministero dell'Università e della Ricerca Scientifica e Tecnologica, Roma, from the Fondazione IRCCS Policlinico S. Matteo, Pavia, and from the 'Ferrata-Storti Foundation', Pavia.

\section{References}

1. Connors JM: Evolving approaches to primary treatment of Hodgkin's lymphoma. Hematology Am Soc Hematol Educ Program 2005: 239-244, 2005

2. Federico M, Luminari S, Iannitto E, et al: ABVD vs. BEACOPP vs. CEC for the initial treatment of patients with advanced Hodkin lymphoma. Results from the HD2000 GISL Trial. J Clin Oncol (In press).
3. Klimm B, Schnell R, Diehl V and Engert A: Current treatment and immunotherapy of Hodgkin's lymphoma. Haematologica 90: 1680-1692, 2005.

4. Bonadonna G, Zucali R, Monfardini S, De Lena M and Uslenghi C: Combination chemotherapy of Hodgkin's disease with adriamycin, bleomycin, vinblastine, and imidazole carboxamide versus MOPP. Cancer 36: 252-259, 1975.

5. Gobbi PG, Broglia C, Levis A, et al: MOPPEBVCAD chemotherapy with limited and conditioned radiotherapy in advanced Hodgkin's lymphoma: 10-year results, late toxicity and second tumors. Clin Cancer Res 12: 529-535, 2006.

6. Diehl V, Franklin J, Pfreundschhuh M, et al: Standard and increased-dose BEACOPP chemotherapy compared with COPP-ABVD for advanced Hodgkin's disease. N Engl J Med 348: 2386-2395, 2003

7. Armitage P and Berry G: Statistical methods in medical research. 2nd edition, Blackwell Scientific Publications, Oxford, pp 408-417. 1987.

8. Houghton JA: Apoptosis and drug response. Curr Opin Oncol 11: 475-481, 1999

9. Bartlett NL: Therapies for relapsed Hodgkin's lymphoma: transplant and non-transplant approaches including immunotherapy. Hematology Am Soc Hematol Educ Program pp245$251,2005$.

10. Federico M, Luminari S, Dell'Olio M, et al: ABVD vs. COPPEBVCAD (CEC) vs. BEACOPP for the initial treatment of patients with advanced Hodgkin's lymphoma (HL). Preliminary results of HD2000 trial. Haematologica 92 (Suppl 5): Abst. C026, 34, 2007.

11. Danova M, Rosti V and Mazzini G: Cell kinetics of CD34positive hematopoietic cells following chemotherapy plus colony-stimulating factors in advanced breast cancer. Int $\mathrm{J}$ Cancer 63: 646-651, 1995. 